\title{
LA REVITALIZACIÓN DE ESPACIOS PÚBLICOS DOTA- CIONALES EN LA CIUDAD DE MADRID
}

\author{
Amaya CASADO ECHARREN \\ Ayuntamiento de Madrid \\ Departamento de Derecho Administrativo. Universidad Nacional de Educación \\ a Distancia (UNED) \\ amayacasado@der.uned.es
}

\author{
Marta LORA-TAMAYO VALLVÉ \\ Departamento de Derecho Administrativo. Universidad Nacional de Educación \\ a Distancia (UNED) \\ mlorat@der.uned.es
}

Recibido: 30/09/2013

Aceptado: 10/12/2013

RESUMEN: El presente trabajo se centra en el análisis y la reflexión sobre el estado en el que se encuentran en el momento actual, las actuaciones de revitalización de espacios públicos dotacionales en la ciudad de Madrid. Para ello, y dentro de las actuaciones de revitalización de espacios públicos contempladas en el Plan de Acción del Área de Gobierno de Urbanismo y Vivienda para la Revitalización del Centro Urbano, se han seleccionado dos operaciones muy significativas tanto por su localización como por el impacto que las mismas han producido en la imagen del centro urbano de la ciudad. Se trata de la actuación en el ámbito del Mercado de Barceló y en el ámbito del Mercado de la Cebada. Ambas actuaciones comparten un elemento común que es el de la intervención sobre un Mercado Municipal, que de ser un espacio en franca degradación y retroceso, por el cambio de hábitos de consumo de la población del centro ha pasado a convertirse en un factor de dinamización y potenciación de la actividad económica, social y cultural... Estos procesos no están exentos, sin embargo, de ciertas problemáticas derivadas básicamente de la disposición de los suelos dotacionales públicos para su conversión en usos lucrativos que permitan financiar y por lo tanto asegurar el éxito de las actuaciones, y de la necesidad de garantizar la calidad y la cantidad de las nuevas dotaciones compensatorias.

1 El presente texto se incardina en el proyecto de investigación Las ciudades españolas en la etapa autonómica (1978-2012). Dinámicas, procesos y políticas (URBSPAIN), financiado por el Plan Nacional de Investigación I+D+i del Ministerio de Economía y Competitividad para el período 2010-2013 (Código CSO 2009-11261-Subpr. GEOG). 
PALABRAS CLAVE: Actuación de revitalización urbana. Espacios públicos dotacionales. Centro Urbano. Mercados Municipales.

\section{REVITALIZATION OF PUBLIC SP ACE FOR COMMUNITY USE IN THE CITY OF MADRID}

ABSTRACT: This work focuses on the analysis and reflection on the state you are in the present moment, the actions of revitalizing public spaces dotacionales in the city of Madrid. For this, and in the actions of revitalizing public spaces in the Plan of Action of the Government Department of Planning and Housing for the Urban Center Revitalization, we selected two very significant both for its location and the impact that the same occurred in the image of the center of the city. This is the action in the field Barceló Market and within the scope of the Barley Market. Both actions share a common element of the intervention is on a municipal market, that of being an open space and reverse degradation, by changing consumption habits of the population of the center has gone on to become a factor of dynamism and enhancement of economic activity, social and cultural... These processes are not free, however, certain problems arising mainly from the provision of public dotacionales soils for conversion into profitable uses for financing and thus ensure the success of the actions, and the need to ensure quality and the amount of new allocations compensatory.

KEYWORDS: Performance of urban revitalization. Dotacionales Public Spaces. Urban Center. Municipal Markets.

\section{INTRODUCCIÓN Y ESTADO DE LA CUESTIÓN.}

En los últimos años estamos asistiendo a un evidente cambio del paradigma en las políticas públicas territoriales y urbanas de las grandes ciudades en el territorio español. Así y tras un ciclo de políticas urbanas expansionistas de creación y crecimiento de los núcleos urbanos, mediante su ensanche y prolongación de los mismos, aparecen con fuerza nuevos planteamientos enfocados en la conservación y recuperación de los cascos urbanos.

Dos son las causas que pueden orientar sobre este cambio de estrategia y explicar la opción de los poderes públicos de reconsiderar las políticas urbanas para la reformulación y replanteamiento de nuevos objetivos y actuaciones, a saber, la situación de deterioro y abandono de ámbitos urbanos ya consolidados, así como el impacto de la crisis económica en el proceso de creación de nueva ciudad.

Ciertamente, se detecta una problemática, perfectamente predicable de la mayor parte de los cascos urbanos de los grandes municipios, que afecta sobre todo a los Cascos Históricos con graves deficiencias de rehabilitación, a polígonos de viviendas con antigüedad superior a los 40 años y a Áreas Urbanas surgidas como procesos de urbanización marginales (HeRvás, 2009). Esta problemática que conlleva un deterioro físico y social de los cascos urbanos, que se plasma en aspectos tales como: 
- Obsolescencia de la urbanización de ámbitos localizados en el centro de la ciudad e infradotación de los servicios urbanísticos.

- Proliferación de la infravivienda.

- Déficit de equipamientos, zonas verdes, espacios urbanos de calidad.

- Generación y potenciación de efectos sociales negativos: envejecimiento de la población residente, paulatino desplazamiento de la población joven hacia la periferia, ocupación de estas áreas marginales por sectores poblaciones con escasos recursos económicos con pautas de comportamiento muy diferenciadas a las de la población residente en origen, que generan importantes problemas de marginalidad e integración social

- Aislamiento de estos ámbitos con el entorno, por ausencia de elementos que les permitan ser un reclamo para población del entorno (ausencia de actividad comercial, cultural, social, etc..) y surgimiento de formas de actividad económica ilegal.

Estas circunstancias son un claro signo de decadencia de ciertos ámbitos urbanos y de un proceso degenerativo y de descuido que a la postre confluyen en el abandono de los espacios públicos y, con el tiempo, de los espacios concernientes a las propias Comunidades de propietarios, a lo que también hay que sumar el impacto negativo, por contagio, que estas situaciones provocan en entornos urbanos colindantes.

Centrándonos en el caso de la ciudad de Madrid, resulta curioso constatar cómo el planificador de 1997, a pesar de estar en pleno auge las políticas expansionistas de crecimiento de la ciudad, ya fijó entre los objetivos del Plan General de Ordenación Urbana los propios de las políticas urbanas de recuperación y revitalización del centro urbano, aun cuando las mismas, por aquel entonces, estaban motivadas más en la protección del patrimonio histórico artístico del centro de la ciudad que en la necesidad de buscar fórmulas alternativas de hacer ciudad.

Así, comprobamos cómo el Plan General de Ordenación Urbana de Madrid de 1997 actualmente vigente, supuso una concepción novedosa en el tratamiento y la forma de abordar tanto el patrimonio edificado de interés histórico-artístico como la recuperación y revitalización del Casco Histórico, identificado territorialmente con la "almendra central de Madrid".

De un planteamiento meramente proteccionista y conservador de los elementos configuradotes y definidores del centro de la ciudad, propios de la filosofía "prohibicionista" de los años 50 y 60, dirigido a evitar la destrucción del 
patrimonio urbano, el PGOUM asume un planteamiento positivista y posibilista dirigido a la puesta en valor de los valores y rasgos propios del centro de la ciudad combinando de forma efectiva y equilibrada la modernización y actualización de los entornos y su recuperación y revitalización.

En coherencia con este nuevo concepto de ordenación del centro histórico de la ciudad, el PGOUM incluyó dentro de las metas a conseguir la Revitalización de la ciudad consolidada, especialmente del Casco Antiguo y el Centro Histórico, compaginando los procesos de conservación y cambio y protegiendo la estructura urbana y edificatoria de las áreas de mayor valor arquitectónico, histórico y medioambiental. Esta idea responde al principio de flexibilidad frente al sobredeterminismo que inspiró la redacción del PGOUM anterior y que pretende superar las tensiones que se producían entre la realidad cambiante y dinámica de la ciudad y las determinaciones, reglas y limitaciones excesivamente rígidas que imponía el planeamiento precedente haciendo imposible cualquier intento de innovación, modernización y respuesta ante nuevas demandas. En este mismo sentido, el PGOUM apostó por propiciar y posibilitar las nuevas alternativas urbanísticas fruto de la dinámica de una ciudad abierta y moderna equilibrando las el máximo rendimiento de las distintas alternativas con medidas de control convenientes.

Dentro de este marco de intenciones, el PGOUM de 1997 marcó una serie de objetivos con impacto directo en las políticas de "Espacios Públicos". En concreto estos objetivos, definidos en el documento de Memora del PGOUM de 1997, fueron:

1.- Mejora de la movilidad y en especial de la accesibilidad.

La realización de múltiples actividades, los contactos entre personas, el acceso a los bienes culturales, de recreo, de consumo, la distribución de materias de todo tipo, requieren de espacios urbanos adecuados desde el punto de vista de la movilidad y la accesibilidad.

El conjunto integrado por la red viaria y el sistema de transportes constituye un instrumento esencial de la ordenación del territorio y del desarrollo urbano y tienen una influencia fundamental tanto en las comunicaciones con el exterior como en el apoyo a las estrategias de localización de actividades económicas y residenciales. Su importancia se manifiesta como instrumento de integración espacial y vertebración de los distintos sectores, así como agente básico en la calidad de vida urbana y por los impactos ambientales que las infraestructuras y los vehículos ocasionan sobre el medio natural y urbano. Por todo ello, el PGOUM apostó por: 
- Un modelo de transporte colectivo basado en la integración del ferrocarril y el metro en el contexto de un sistema multimodal integrado, aumentado su oferta.

- Acceso y recuperación de nuevas áreas de oportunidad o focos de atracción, que favorezcan una combinación de usos y actividades.

- Favorecer el tránsito peatonal, disminuyendo el conflicto entre el vehículo privado y el peatón a través de al creación de itinerarios peatonales en relación con la creación de equipamientos y usos terciarios comerciales

- Compatibilización del desarrollo de las infraestructuras de transporte con el medio ambiente.

2.- Establecer un modelo de ciudad equilibrada a través de los equipamientos y los espacios libres.

La premisa de partida era la congestión existente el áreas centrales, derivada de la elevada densidad poblacional y de la concentración de usos terciarios agravada por la falta de zonas de esparcimiento, deterioro de los espacios públicos urbanos y ausencia de instalaciones deportivas. Todo ello planteó como objetivo la recuperación y puesta en valor de los equipamientos en el centro urbano y de los espacios públicos y sus zonas verdes, potenciándolos como lugares para el desarrollo de actividades lúdicas y simbólicas de la colectividad. Asimismo, se determinó la necesidad de reforzar la consideración de estos espacios libres como verdadero patrimonio y servicio público convirtiéndolos en un instrumento atractivo para la creación de un modelo de ciudad equilibrada. La necesaria integración de las zonas verdes y los espacios libres en la estructura urbana precisaba de la definición de un sistema continuo de toda la trama que definiera aquélla, que sirviera de enlace ininterrumpido entre todos los elementos, en definitiva una sucesión de espacios libres con dedicación al ocio, al esparcimiento que permitieran acentuar la variedad urbana, reducir la densidad, potenciar los desplazamientos peatonales y el uso de los espacios libres existentes.

El equipamiento se pasa a concebir como soporte y catalizador de las relaciones sociales, para favorecer la vertebración de la vida social en los barrios madrileños, fomentando la participación de los distintos grupos sociales, diversificando la oferta de espacios para usos dotacionales. Se busca flexibilizar el uso concreto de los equipamientos locales para su adaptación a las necesidades básicas del barrio, tratando de compatibilizar estos equipamientos locales con los equipamientos singulares. 


\section{3.- Revitalización de centro Histórico de la Ciudad.}

Se conceptualizan los espacios públicos del centro de la ciudad como ámbitos dónde se sedimentan los mejores valores de la ciudad y, a la vez, los mayores problemas. Por ello el PGOUM planteó un tratamiento específico e individualizado para el centro urbano de la ciudad, al objeto de salvaguardar sus valores, reformar su tejido, y crear condiciones idóneas para su regeneración simbólica, funcional y social. Así el PGOUM proyectó tanto en el Centro Urbano de la ciudad como en el Casco Antiguo una serie de operaciones de regeneración del tejido urbano, cuyo motor debía ser en esencia la iniciativa pública. Como síntesis de esta meta, la Memoria del PGOUM identificó los siguientes objetivos concretos para el centro urbano:

- Potenciar el carácter residencial del centro garantizando el nivel de servicios adecuado.

- Corregir la tendencia a la terciarización indiscriminada

- Optimización del transporte colectivo

- Previsión de nuevos espacios libres y equipamientos

- Selección de equipamientos que permitan cualificar el carácter emblemático y simbólico del centro.

- Recuperar la calidad ambiental mejorando la imagen urbana y adecuando su morfología.

Para la concreción y desarrollo plausible de los objetivos marcados en la Memoria del PGOU, se aprobó el Plan de Acción del Área de Gobierno de Urbanismo y Vivienda para la Revitalización del Centro Urbano, con la pretensión de obtener un centro urbano sostenible mediante el desarrollo de objetivos como:

- La promoción del uso residencial, mediante la incorporación de los jóvenes como residentes, la desaparición de infravivienda y la generación de nuevos modelos de vivienda.

- La promoción de actividades económicas y comerciales que colaborasen en fijar la residencia.

- El desarrollo del bienestar social, incrementando el nivel de equipamientos y favoreciendo la integración social, mejorando el medio ambiente urbano, consolidando la "centralidad cultural" como elemento identificativo del Centro de Madrid, transformando el paisaje urbano, creando una nueva "imagen del Centro", mediante el diseño de los espacios públicos. 
Este Plan fue presentado el año 2004, y ha tenido su desarrollo a lo largo de los últimos años, en sus diferentes capítulos: Plan de Promoción del Uso Residencial, Programa de Acciones para la Rehabilitación Residencial y Plan de Acciones para el Desarrollo Dotacional.

Centrándonos en el apartado de Acciones para el Desarrollo Dotacional, en coherencia con el tema que se aborda en el presente trabajo, la acción municipal se ha centrado en diversos proyectos:

- Plan Director de Recuperación de Bulevares

- Plan Director Delicias-Méndez Álvaro-Abroñigal

- Intercambiador de Legazpi

- Modificación del PGOUM 97 para la remodelación del

- Museo Arqueológico Nacional

- Modificación del PGOUM 97 en la plaza de la Lealtad 5 (Hotel Ritz)

- Plan Especial de protección del Real Jardín Botánico de Madrid

- Plan Especial de protección, cambio de uso y ampliación del edificio de Joaquín María López 61

- Plan Especial de adaptación, mejora y ampliación de equipamientos en la parcela municipal sita en la calle Doctor Fourquet 22-24

- Actuación en el ámbito del Mercado y la calle de Barceló

- Revitalización de la plaza de la Cebada

- Mercado de Frutas y Verduras

Todos los antecedentes expuestos permiten, en consecuencia, acudir experimentalmente a la ciudad de Madrid, como un importante exponente de gran ciudad que tras las crisis económica que ha eclosionado tras la "burbuja inmobiliaria" ha optado por potenciar y desarrollar actuaciones dirigidas a la regeneración y revitalización de los espacios públicos como mecanismos para la reactivación social, económica, cultural, medioambiental y patrimonial de los mismos.

No obstante, se verá cómo paradójicamente la misma crisis económica que ha centrado la atención de los poderes públicos municipales en la recuperación y puesta en valor de los espacios públicos del centro de la ciudad, ha sido responsable del ritmo de ejecución e incluso del éxito último de las mismas. Esta circunstancia también a propiciado la tentativa por parte del Ayuntamiento de nuevas fórmulas de gestión y ejecución, que en última instancia han encontrado eco en la reciente normativa estatal en materia de Rehabilitación, regeneración y reforma Urbana (Ley 8/2013 de 26 de junio) 


\section{METODOLOGÍA, FUENTES, HIPÓTESIS DE TRABAJO.}

En el presente trabajo se ha optado por realizar una valoración del conjunto de actuaciones de revitalización de espacios dotacionales, previstos en el marco del Plan de Acción del Área de Gobierno de Urbanismo y Vivienda para la Revitalización del Centro Urbano de 2004, con la finalidad de valorar la incidencia que sobre ellos ha tenido la situación de crisis económica global y específica en el sector inmobiliario.

A tales efectos, y en función de los datos que sobre el ritmo de ejecución y culminación de las mismas ofrecen los documentos de Memorias de Gestión del Área de Gobierno de Urbanismo y Vivienda, correspondientes a los años 20082009 y 2010, cabe hacer una primera reflexión en relación con estas acciones para el desarrollo dotacional y es que a fecha de hoy sólo se han culminado aquellas cuya ejecución estaba prevista o muy avanzada en el año 2008, momento en el que se pusieron de manifiesto de un modo más palmario los efectos de la crisis económica y a los que desde luego no fue ajena la Administración Municipal, que ha visto reducidas de manera relevante sus posibilidades de inversión económica en actuaciones de iniciativa y gestión pública.

Con ello se constata sin dificultad, cómo la situación de crisis económica ha agravado, sino impedido, las posibilidades de desarrollo tanto de las operaciones de creación de ciudad como las de recuperación urbana, generando un contexto desfavorable para la inversión pública, ceñida a severos procesos de estabilización presupuestaria y la financiación económica sometida a importantes restricciones en el acceso al crédito.

Bajo tales condicionantes se han podido identificar tres tipos de situaciones:

\section{1.- Actuaciones de revitalización dotacional culminadas.}

Exponente de estas son las Escuelas Pías de san Antón. El objetivo de esta actuación era rehabilitar el edificio de las antiguas Escuelas Pías, mediante la implantación de nuevas dotaciones, destinadas a mejorar la calidad de vida del vecindario y la consolidación del centro de la ciudad como un espacio atractivo y habitable. Para la consecución de este objetivo en el año 2006, el Ayuntamiento de Madrid suscribió un Convenio de Colaboración con el Colegio de Arquitectos de Madrid, para la rehabilitación del antiguo Colegio de San Antón como nueva sede del Colegio Oficial de Arquitectos de Madrid (COAM), así como para la incorporación de un conjunto de nuevos equipamientos municipales.

Mediante dicho acuerdo, el COAM asumía el coste de rehabilitación del edificio y el Ayuntamiento cedía, a través de un derecho de superficie por un pe- 
riodo de 75 años, un total de $12.000 \mathrm{~m} 2$ de $\operatorname{los} 21.000 \mathrm{~m} 2$ que corresponden a la edificabilidad estimada como capacidad máxima del edificio. Los restantes 9.000 $\mathrm{m} 2$ se destinaban al programa de equipamientos (Escuela Infantil, Centro de Día, Centro de Mayores y Equipamiento Deportivo que se disponen como remate del Instituto existente y del Colegio de Farmacéuticos orientados al jardín arbolado.) Con objeto de aprovechar la capacidad edificatoria del ámbito, el acuerdo contemplaba también la construcción de un aparcamiento con una capacidad estimada de 500 plazas, de las que dos tercios (2/3) se destinarán a aparcamiento de residentes.

\section{2.- Actuaciones de revitalización dotacional no culminadas.}

Se trata de un significativo número de actuaciones respecto de las cuales las propias Memorias de Gestión señalan que los trabajos no ha podido culminarse al haberse suspendido temporalmente el contrato por decisión municipal, debido a la coyuntura económica y a la falta de actualización de recursos propios de las Haciendas Locales. Por lo que, aún siendo la redacción del estos planes documentos de trabajo importantes para la regeneración integral del espacio libre público en la ciudad, se ha remitido su redacción final a un futuro próximo.

Por citar algún ejemplo, se encuentran en esta situación el Plan Director de Recuperación de los Bulevares, con el que se pretendía mejorar los espacios públicos destinados al tránsito peatonal y a la estancia en los viarios que estructuran la Almendra Central, o el Plan Director Delicias-Méndez Álvaro, concebido para dar respuesta a los problemas urbanos de conexión, accesibilidad, de definición de usos y actividades, así como de dotaciones singulares y locales que presenta el ámbito en cuestión y que hacían necesario la mejora de las Infraestructuras ferroviarias de alta velocidad y la ciudad así como su terciarización del ámbito

\section{3.- Actuaciones en desarrollo.}

En este grupo se insertan todas las actuaciones de revitalización de espacios urbanos públicos que se encuentran tanto en tramitación como en ejecución. En concreto el análisis efectuado versa sobre las actuaciones en:

a) Mercado Barceló

b) Mercado de la Cebada. APE 01.07 "Plaza de la Cebada-Carrera de San Francisco". Distrito centro.

Ambas presentan una serie de rasgos definidores que ilustran muy bien la situación en la que se encuentran actualmente las actuaciones de revitalización del centro urbano de Madrid, y que bien podrían sintetizarse en que: 
- Su ejecución se vincula con la recuperación y mejora global de ámbitos de suelo urbano consolidado localizados en el centro de la ciudad.

- Afectan a ámbitos de titularidad pública.

- Están planificadas como actuaciones de iniciativa pública y basadas, al menos en su concepción inicial en la financiación pública.

- Representan un cambio en el modelo municipal de calidad urbana, que pasa de la puesta por potenciar los usos dotacionales a incorporar, combinadamente, usos lucrativos.

- Su ritmo de ejecución se ha visto condicionado, directamente, por el impacto de la crisis económica y financiera.

- Incorporan nuevas fórmulas para garantizar su viabilidad económica, que pasan por incorporar a la iniciativa privada.

La reflexión sobre todos estos factores, permiten aventurar que la financiación se ha convertido en el eje central y el aspecto clave de todas las políticas públicas en materia de revitalización y regeneración urbana en la ciudad de Madrid, de cuya previsión dependerá, en gran medida, el éxito o el fracaso de las actuaciones proyectadas. El tema de la financiación de las operaciones de rehabilitación y regeneración urbana, ya ha sido objeto de interés por parte del legislador urbanístico desde principios de esta década, consciente de las dificultades económicas y del enorme coste que todos estos procesos comportan para las respectivas administraciones Públicas. Así, la Comunidad de Madrid dedica el 2\% del presupuesto de la Consejería competente en materia de Obras Públicas y Vivienda a la ejecución de estos procesos de resuperación urbana, al tiempo que ha regulado, en el artículo 12 de la Ley 7/2000 de Rehabilitación de Espacios Urbanos de Madrid, un régimen jurídico específico para los Programas de Rehabilitación Concertada. Por su parte, en Cataluña existe un Fondo Financiero específico para la rehabilitación de áreas degradadas, con el que se han financiado 92 actuaciones. En Valencia el Decreto 76/2007, de 18 de mayo, por el que se aprueba el reglamento de Edificios y viviendas. En Aragón, Decreto 225/2005 de 2 de noviembre, regulador del plan para facilitar el acceso a la vivienda y fomentar la rehabilitación 2005-2009. Finalmente encontramos el Decreto 317/2002 de 230 de diciembre, sobre actuaciones protegidas del patrimonio urbanizado y edificado del país Vasco. Por parte del Estado, y para el periodo 2009-2012 se aprobó el Plan Estatal de Vivienda por Real Decreto 2066/2008 de 12 de diciembre, en el que se contemplaban una serie de subvenciones y ayudas públicas del Ministerio de vivienda para la rehabilitación de edificios, viviendas, obras de ur- 
banización y reurbanización, obras de mejora de la habitabilidad, accesibilidad, etc.

En el marco jurídico actual, hay que destacar la reciente Ley 8/2013 de 26 de junio, de rehabilitación, regeneración y renovación urbanas. Se parte en esta regulación de la superación de la legislación urbanística aprobada hasta la fecha con el objetivo básico de regular los mecanismos oportunos para la creación de la ciudad para dar paso a las actuaciones de recuperación y puesta en valor de los tejidos urbanos existentes, articulando fórmulas innovadoras, creativas e inteligentes para el fomento del bienestar social, económico y de la calidad de vida de los ciudadanos. Se pretende generar un marco normativo idóneo que cubra ciertas lagunas y elimine algunos obstáculos en materia de revitalziación, para lo que introduce instrumentos de gestión y mecanismos de cooperación interadministrativa que tienen por objeto fortalecer el marco en el que se desarrollan estas actuaciones y se establecen mecanismos específicos para facilitar la financiación de estas actuaciones (explotación conjunta de los inmuebles, contratos de cesión de arrendamiento, de otorgamiento de derecho de explotación a terceros, permutas, consorcios, sociedades mixtas...).

\section{ESTUDIO EMPÍRICO}

Para la realización del estudio empírico de las actuaciones seleccionadas, se parte del análisis de los distintos expedientes administrativos, de manera conjunta y comparada, en los que se definen cada una de ellas, con la identificación de los objetivos propuestos y la referencia a los siguientes aspectos más significativos:

a) Sus antecedentes: ámbito de actuación, justificación de la operación, objetivos propuestos y opciones para su ejecución.

b) El instrumento de planeamiento elegido para la ordenación pormenorizada de los ámbitos.

c) Estudio de viabilidad económico-financiera de la actuación

a) Antecedentes.

La premisa de partida de la actuación desarrollada en el ámbito del Mercado de Barceló, es plenamente coherente con los objetivos definidos por la Memoria del PGOUM para la revitalización del Centro a los que ya nos hemos referido. 
De este modo la actuación se justifica en la apuesta urbanística del Ayuntamiento de Madrid por la calidad, recualificación y mejora medioambiental de las zonas más deterioradas del centro histórico de Madrid, para dar respuesta adecuada a las exigencias del desarrollo social, económico y tecnológico. Esta actuación se inserta en el nuevo modelo estratégico para el centro urbano de Madrid, dirigido a rescatar y estructurar nuevos ámbitos de reforma urbana.

El PGOUM delimitó ya el ámbito, de suelo urbano consolidado, configurado por la calle Barceló, el Mercado de Barceló y la zona de los jardines del arquitecto Ribera, como Área de Planeamiento Específico 01.03 "Barceló", previendo los siguientes objetivos para el mismo: "Optimizar el funcionamiento del mercado resolviendo la carga y descargar, construir un polideportivo de uso público compartido con el Instituto, ampliar las superficies viarias de usos peatonales en las calles Barceló y Beneficiencia y potenciar la actividad de la zona creando un centro artesanal en el actual edificio Senpa"

Tras el análisis concreto del ámbito y de la capacidad potencial del tejido urbano, se decidió tramitar una Modificación Puntual del PGOUM (aprobada definitivamente mediante Orden del Consejero de Medio Ambiente y Ordenación del Territorio de la Comunidad de Madrid de fecha 20 de julio de 2006), con al finalidad de corregir los déficit dotacionales del ámbito, el propio deterioro del Mercado así como para acometer una rehabilitación más profunda y de mayor alcance, incorporando a la actuación la Plaza de Santa Bárbara. Se trata de un ámbito final de $63.392 \mathrm{~m} 2$, de titularidad pública que incorpora suelo calificado a Servicio Público Básico (Mercado Municipal de Barceló), Zona Verde Básica, Deportivo Básico, Equipamiento Básico (Biblioteca pública-cultural) y parcela para Servicios Terciarios de Comercio.

En este marco el Ayuntamiento de Madrid optó por dar cabida a la iniciativa privada, mediante la convocatoria de un concurso de ideas para la reestructuración, urbanización y construcción de edificios dotacionales, que fue resuelto en diciembre de 2008.

En el caso de la actuación seguida en el Mercado de la Cebada el objetivo era la recuperación y la mejora ambiental de un ámbito de titularidad pública de 17. $900 \mathrm{~m} 2$ ubicado en pleno Centro Histórico de Madrid, para adaptarlo a las nuevas condiciones de desarrollo social, ambiental, económico, cultural, económico, tecnológico pero manteniendo un adecuado equilibrio con las condiciones históricas y ambientales del ámbito.

Para su consecución también se ha seguido la fórmula de tramitar una Modificación Puntual del Plan General de Ordenación Urbana de Madrid que esta- 
blezca el marco general adecuado para acometer lo que el propio Plan General denomina como una operación de reforma y renovación integral de la ordenación y urbanización del ámbito consistente en la sustitución de parte del uso dotacional de servicios colectivos (categoría abastecimiento alimentario) del Mercado de la Cebada por el de servicios terciarios (gran superficie comercial) y, en consecuencia en articular medidas compensatorias del aumento de la edificabilidad terciaria en el edificio del Mercado de la Cebada, aumentando para ello la superficie del uso dotacional, de las zonas verdes y del uso deportivo.

b) Figura de planeamiento elegida para la ordenación pormenorizada de los ámbitos

Para la ordenación pormenorizada del ámbito del Mercado de Barceló, el propio PGOUM opta por la figura del plan especial, dotado de un amplio margen de maniobra a partir de la regulación amplia que de ellos hizo el artículo 50 de la Ley 9/2001, de 17 de julio, de Suelo de la Comunidad de Madrid. Así el apartado 2 del citado artículo 50 LSCM dispone que "El Plan Especial podrá modificar o mejorar la ordenación pormenorizada previamente establecida por cualquier otra figura de planeamiento urbanistico debiendo justificar suficientemente en cualquier caso su coherencia con la ordenación estructurante".

Se tramita, por tanto, un Plan Especial de definición y mejora de la ordenación pormenorizada de redes públicas locales y control urbanístico-ambiental de los usos, justificado en la necesidad de ofrecer viabilidad urbanística al proyecto resultante del concurso de ideas y estableciendo una ordenación basada en los siguientes aspectos: demolición del edificio del antiguo mercado de Barceló y su sustitución por un nuevo y más moderno edificio con el mismo uso y destino, adecuándose en cuanto a su concepción y espacios a las nuevas exigencias técnicas comerciales; liberación de más suelo dotacional y espacios libres, dando lugar a un ámbito formalmente distinto y funcionalmente más dotado e integrado; peatonalización de viario circundante; edificación de un nuevo equipamiento público (biblioteca) y deportivo público y calificación de más zonas verdes; nuevo edificio de uso terciario comercial; previsión de aparcamientos para dar ser servicio al nuevo equipamiento, al mercado y al edificio terciario.

Desde el punto de vista de la protección del patrimonio, el Plan Espacial fue sometido a dictamen de la Comisión de Protección de Patrimonio HistóricoArtístico, quién lo informó favorablemente. En cuanto a los aspectos medioambientales de la actuación, el Plan Especial incorpora el control ambiental de los usos, delimitando la incidencia de los mismos en el medio ambiente urbano (ruido, vibraciones, luminosidad, emisiones, gases, tráficos, plazas de aparcamiento, etc....), estableciendo las medidas correctoras pertinentes para garantizar impac- 
tos negativos antes inexistentes. Los impactos que en este sentido se evalúan son: la levada concentración de personas y vehículos en las horas de uso público de los edificios del Mercado, Polideportivo y biblioteca; concentración de vehículos en las labores de carga y descarga del Mercado; vertidos de aguas residuales y producción de residuos sólidos urbanos.

En el caso de la actuación del Mercado de la Cebada se opta, igualmente, por articular y concretar la ordenación pormenorizada a través de un Plan Especial que, en este caso, denomina de Mejora Urbana, pero que es reconducible igualmente a los planes especiales del artículo 50 de la Ley del Suelo de la Comunidad de Madrid. Pero si bien la opción del Plan especial es una opción bastante convencional, lo que resulta más significativo es que también en este caso, el Plan General optó por convocar un Concurso de Ideas sobre las distintas propuestas finales de diseño, a cuyo resultado debía adaptarse la ordenación pormenorizada del Plan Especial que finalmente contempló los equipamientos y servicios públicos (polideportivo municipal; gimnasios, pistas polivalentes, piscinas, incluye zona para uso del Colegio Público La Paloma) del ámbito; las superficies asignadas a los distintos usos, la localización de estos usos; la construcción de un aparcamiento bajo rasante, al servicio del uso terciario comercial a implantar en el Mercado y, finalmente la definición de la urbanización y el ajardinamiento del ámbito.

\section{c) Estudio de viabilidad económico-financiera de la actuación.}

El estudio de viabilidad económica de las operaciones de regeneración o revitalización urbanas es una exigencia que deriva del artículo 43. b) de la Ley 9/2001, de 17 de julio de suelo de la Comunidad de Madrid, y que se erige, en definitiva como la pieza clave de la viabilidad de cada actuación al medir, en términos objetivos, reales y tangibles, la posibilidades de realización efectiva de la actuación. Sobre el estudio económico-financiero, resulta interesante comprobar cómo la nueva regulación estatal sobre las actuaciones de regeneración, rehabilitación y reforma urbana, establece su exigencia como elemento central e ineludible de las mismas vinculado al concepto de sostenibilidad. Así, la Ley 8/2013 en su Disposición Final 12 modifica el artículo 15.4 TRLS 2008 para incorporar expresamente la exigencia del informe o memoria de sostenibilidad económica, en el que se ponderará el impacto de la actuación en la hacienda de la Administración Pública implicada, así como la suficiencia y adecuación del suelo destinado a usos productivos.

Constatamos como en ambos casos la viabilidad económico-financiera de la actuación de revitalización urbana parte de la premisa de asegurar un equilibrio adecuado entre la capacidad del sector público para financiar las cargas económicas derivadas de la obtención de suelo, la urbanización y la edificación que 
corresponde por el planeamiento a la iniciativa pública municipal y los efectos económicos que las operaciones urbanísticas tienen sobre los ámbitos territoriales concretos (revalorizaciones, incrementos de rentas por mejoras urbanas...).

Para ello y en ambas actuaciones, la merma de la capacidad del sector público para la financiación de la operación de renovación urbana, por las especiales circunstancias económicas por las que atraviesa el Ayuntamiento de Madrid, plantean la necesidad de acudir a nuevas fórmulas que permitan a la inversión pública asumir el coste de las mismas. Con este planteamiento el estudio de viabilidad económico-financiera efectuado en ambas actuaciones procede al cálculo de los costes que tiene para el Ayuntamiento la actuación (construcción del Mercado, construcción del equipamiento, construcción del garaje-aparcamiento; ejecución de dotaciones y de obras de urbanización y cálculo de las indemnizaciones por realojo de los comerciantes del marcado público) para determinar, en consecuencia, la edificabilidad lucrativa que es preciso localizar en el ámbito para dar cabida a la inversión privada.

De forma específica en la operación del Mercado de Barceló el estudio económico-financiero refiere como parte decisiva para poder transformar la ciudad, recualificando y revitalizando su Centro Urbano, especialmente aquellas áreas obsoletas y deterioradas de escaso interés y utilización por los ciudadanos, la viabilidad económica de las operaciones revitalizadoras reconociendo que si bien algunas podrán ser sufragadas por los presupuestos municipales, otras, dada su envergadura, deberán poder sufragarse por sí mismas. Por ello el Ayuntamiento, en este caso, consideró la necesidad de poder destinar los ingresos derivados de la enajenación de bienes, concesiones administrativas o alquiler de plazas de aparcamiento derivadas de la ejecución de la operación de recualificación y revitalziación urbana a sufragar la misma, esgrimiendo para ello, al fundamento legal del artículo 176 de la LSCM relativo a la enajenación de los bienes patrimoniales del Patrimonio Municipal del suelo, previa desafectación de los bienes al dominio público.

En el caso del Mercado de la Cebada el estudio de viabilidad económica, asume, como no podía ser de otra manera, la necesidad de garantizar de manera adecuada la sostenibilidad del modelo elegido. No obstante, es un claro exponente de cómo cuando en el año 2008 la situación de crisis económica, que de forma inevitable produjo efectos en la programación de inversiones municipales, hizo aconsejable un replanteamiento de la actuación para asegurar su viabilidad, se procedió al estudio de diferentes alternativas, más o menos novedosas, de diseño, gestión y explotación de los edificios del mercado, de las dotaciones y de los aparcamientos, con la intención de optimizar los productos resultantes y hacer viable y rentable la actuación, obteniendo flexibilidad en los usos urbanísticos y en las 
diferentes fórmulas de gestión que garantizando los usos públicos pudieran dar viabilidad económica y financiera a la operación de transformación urbana. Tras la valoración de diferentes alternativas para rediseñar la actuación, se adoptaron una serie de decisiones que desde luego son una buena muestra, como decimos, de la incorporación de figuras/mecanismos previstos en el Ordenamiento jurídico para mantener o poner en marcha procesos de regeneración urbana y reactivación económica, social, cultural, etc. Se adopta un nuevo concepto de Mercado, en el que se diversifica la oferta tradicional de los mercados con una nueva oferta comercial, deportiva, lúdica, recreativa y de ocio que las articule para atender las nuevas necesidades de los ciudadanos. Para ello se sustituye el uso cualificado de la parcela de Servicios Públicos Colectivos, en su clase Servicio Público abastecimiento alimentario, por el de Servicios Terciarios en su clase Comercial, categoría Gran Superficie Comercial, el cual es susceptible de ser enajenado a la propiedad privada tras su desafectación al uso público. Se incorpora la iniciativa privada para que sea el capital privado el que asuma la gestión y explotación del la actividad de Mercado mediante contrato de concesión. Se altera la proporción de los usos previstos, diversificándolos y flexibilizándolos funcionalmente, para mantener el equilibrio entre los dotacionales y las actividades económicas. Si inicialmente se compensaba el paso del Mercado como dotación pública al Mercado como uso terciario, ahora se incrementa la edificabilidad del terciario para resulte rentable a la iniciativa privada y en consecuencia se incrementan los $\mathrm{m}^{2}$ de los usos dotacionales, zonas verdes y viario.. De forma novedosa, se acude a las nuevas figuras de gestión para ámbitos urbanos, aplicando la figura del complejo inmobiliario prevista en el artículo 17 del TRLS 2008.

\section{EVALUACIÓN DE LAS ACTUACIONES ANALIZADAS}

En las actuaciones de revitalización de espacios dotacionales seleccionadas cabe identificar y extraer una serie de factores y elementos comunes que permiten efectuar una valoración global de las mismas.

\section{a) Percepción ciudadana de las actuaciones:}

En el caso de las actuaciones analizadas, las alegaciones formuladas por los particulares y las diferentes asociaciones afectadas por las mismas, ofrecen una información muy valiosa de cara a poder valorar cuáles han sido las principales inquietudes que a nivel, social, urbano, económico, cultural, etc.. han aflorado. Por ello, se recogen las cuestiones de mayor relevancia que fueron alegadas 
en los respectivos trámites de información pública de los expedientes, respecto de las cuales cabe llamar la atención sobre la coincidencia en el fondo de las mismas.

Así, en el caso de la actuación del ámbito del Mercado de Barceló, las alegaciones planteadas por los ciudadanos a la ordenación propuesta se centraron básicamente en los siguientes aspectos:

- Disminución de las dotaciones públicas por la desaparición del equipamiento existente. Este aspecto se contesta por el Ayuntamiento en el sentido de explicar cómo lo que se propicia con la ejecución del Plan Especial es un cambio en la zonificación que por lo tanto afecta a la ubicación del uso del equipamiento preexistente que no desaparece, no pudiéndose hablar de una disminución del uso dotacional del ámbito, que muy al contrario ve incrementada su edificabilidad.

- Privatización parcial de los suelos dotacionales existentes en el ámbito para destinarlos a usos lucrativos no previstos inicialmente en la Modificación Puntual del Plan General. Las alegaciones formuladas en este sentido consideran inaceptables la implantación del uso terciario comercial en un edifico destinado a uso dotacional, así como el resultado que esta decisión produce respecto de la mezcla de usos lucrativos con usos dotacionales. A esta cuestión, el Ayuntamiento señala que el propio Plan General ya admitía en la ficha del Área de Planeamiento Específico el cambio de la ordenación pormenorizada del ámbito para adaptarla a la propuesta ganadora del concurso de ideas en la línea de "mejorar el bienestar, la calidad de vida y promover la humanización de la ciudad". Sobre esta cuestión, y bajo la óptica del criterio de oportunidad, en el informe de contestación a las alegaciones se afirma que "La revitalización de estas zonas, en las que el ciudadano no se encuentra cómodo y por lo tanto, no las usa o no disfruta de ellas, no pasa exclusivamente por la construcción masiva e indiscriminada de equipamientos y zonas verdes. Por eso adquiere tanta importancia en empeño del Ayuntamiento de Madrid, dentro de irrenunciable política de revitalización del Centro Urbano, de potenciar y modernizar otras actividades como son los Ejes Comerciales". Desde el punto de vista del criterio de legalidad, esta desafectación del dominio público, se justifica, en términos jurídicos, con las reglas establecidas en el artículo 8.4 del Reglamento de Bienes de las Entidades Locales. (Decreto de 13 de junio de 1986), de acuerdo con las cuales la Memoria del Plan Especial, supone la alteración de la calificación jurídica del dominio público de forma automática como consecuencia de la aprobación definitiva de 
la modificación del Plan General de Ordenación Urbana de Madrid en el ámbito.

En el caso del Mercado de la Cebada, y en términos similares, constan como principales aspectos alegados los que se refieren a:

- La falta de justificación de las cesiones compensatorias por el incremento de la edificabilidad terciaria al amparo del Artículo 67.2 LSCM, según el cual toda alteración del planeamiento que desafecte suelo de destino público o aumente la edificabilidad deberá establecer medidas compensatorias precisas para mantener la calidad y la calidad de las dotaciones previstas respecto del aprovechamiento urbanístico del suelo, sin incrementar éste en detrimento de la proporción alcanzada entre unas y otro y, en todo caso, asegurar la funcionalidad y el disfrute del sistema de redes de infraestructuras, equipamientos y servicios públicos. Sobre esta alegación, el Ayuntamiento justifica que la actuación de revitalización supone un incremento de la edificabilidad dotacional, inicialmente prevista en el PGOUM, al aumentar las superficies de zona verde, de deportivo y al incorporar una parcela para un nuevo equipamiento público. Asimismo, se justifica el incremento global de los usos dotacionales en el ámbito como consecuencia de la actuación municipal realizada en el proyecto de Madrid-Río. En cuanto a la funcionalidad y calidad de las dotaciones, señala el Ayuntamiento que queda asegurada mediante la integración, a través de un proyecto unitario, de todos los usos dotacionales en torno al nuevo espacio público que se genera en la actuación. Es especialmente relevante, por último, la justificación de que no son precisas medidas compensatorias adicionales para el incremento dotacional que se produce en el ámbito ya que se opta por la figura legal del complejo inmobiliario que mediante la superposición de parcelas en rasante, suelo y vuelo permite localizar todo este incremento en el mismo ámbito.

- La valoración del impacto en el tejido comercial tradicional de la zona y el realojo de los comerciantes concesionarios de los puestos del antiguo Mercado de la Cebada. Se alega que la actuación no garantiza la continuidad de un mercado de barrio, integrado por pequeños comerciantes, que es un valor primordial tanto para la configuración urbana y económica de la ciudad como para la calidad de vida de los vecinos. Sobre esta cuestión se alude, por parte del Ayuntamiento a la elaboración de un estudio de viabilidad económica de la actividad comercial consensuado con los comerciantes afectados a efectos del mantenimiento de su actividad comercial en el nuevo contexto. 
- La desafectación de dominio público para su enajenación a la propiedad privada. Se insiste una vez más en la pérdida de lo público con el consiguiente efecto especulativo al realizarse operaciones de enajenación con fines exclusivamente lucrativos en detrimento del interés general y de los servicios públicos. Se cuestiona, en definitiva el incremento el uso comercial, a costa de lo público, como vía de financiación de la actuación. Esta alternativa se justifica por parte del Ayuntamiento en la posibilidad legal de desafectar bienes de dominio público que pasan a tener la calificación jurídica de bienes patrimoniales los cuales pueden ser enajenados en los términos legalmente previsto.

b) Repercusión de las actuaciones en la imagen de la ciudad.

Se trata de actuaciones localizadas en ámbitos muy concretos de la ciudad y contextualizadas en el centro urbano. No obstante y al margen de estar físicamente acotadas lo cierto es que quedan englobadas en un proceso más amplio de revitalización urbana encauzado a través de una política pública de regeneración muy definida y enmarcada en los objetivos que ya fijó el Plan General de Ordenación Urbana en 1997.

Por ello, y a pesar de ser actuaciones diseminadas y aparentemente fragmentadas, la suma de todas ellas tiene un innegable impacto en la imagen final de la ciudad. Prueba de ello es el Programa Operativo del Área de Gobierno de Urbanismo y Vivienda (2007-2011) el cual recoge como línea de actuación, la revitalización de la almendra central de Madrid, la necesidad de dar continuidad a la línea estratégica iniciada en la legislatura anterior, con el fin de reactivar el potencial del Centro Histórico de Madrid como referente metropolitano y como lugar donde habitar generando nuevos espacios de oportunidad e integrar las diferentes propuestas sectoriales, para conseguir un nuevo florecimiento en la "Almendra Central", en términos de habitabilidad y sostenibilidad en lo cultural, en la integración y en la competitividad. El carácter integral y global de las operaciones de revitalización queda también plasmado en la descripción que el Programa Operativo hace de las acciones concretas a desarrollar y que implican tanto a las áreas municipales como a los distritos a través de un "Plan de Acción Territorial que establezca las directrices de la intervención municipal, con el fin de implantar un modelo integrado, sostenible, de movilidad, mejora del espacio publico y la escena urbana, mejora de las condiciones de implantación de usos y actividades, de la infraestructuras, así como la dotación de más y mejores equipamientos." 
c) Los Mercados municipales como catalizador es de la regeneración urbana.

Pero quizá el elemento más innovador de las operaciones de revitalización urbana seleccionadas sea el de la nueva proyección, dimensión y protagonismo que adquieren los Mercados Municipales, los cuales pasan de ser elementos tradicionales con impacto a nivel de barrio y vecinos, a elementos de modernización con trascendencia global en la ciudad de Madrid, ciudadanos y turistas.

Frente a las cuestiones alegadas a nivel ciudadano, comprobamos la existencia de otra corriente en torno a la redefinición y potenciación de los Mercados Municipales como instrumentos para la recuperación de la ciudad. Esta alternativa parte de que la mayoría de los mercados tienen una localización estratégica al encontrarse en el centro de los barrios, siendo el principal problema la falta de innovación respecto de su actividad que debería adaptarse a la evolución de necesidades y demanda de abastecimiento de los ciudadanos. Este nuevo enfoque, haría compatible la diversificación de servicios y la existencia de supermercados en sus instalaciones. Otro de los aspectos fundamentales que se propugnan desde esta óptica es el de los horarios como forma de adaptarse a las necesidades de consumo del cliente, así como el reenfoque de su actividad al turismo. Como aspecto final se plantea que la gestión de los Mercados municipales sea asumida por empresas externas al Ayuntamiento

\section{d) Aspectos de las actuaciones extrapolables.}

La redefinición de la figura de los Mercados Municipales se convierte en un elemento perfectamente trasladable, como ha desmostrado la experiencia, a otros municipios en los que concurren las mismas necesidades de recuperación de su centro urbano.

Tal es el caso de Barcelona, ciudad en la que ha tenido lugar un proceso importante de modernización y remodelación de los mercados y de impulso de una serie de cambios que buscan unos mercados municipales más competitivos, con instalaciones modernas y los servicios que pide la ciudadanía. Se trata además de un modelo que se está exportando con éxito, tanto a escala nacional como internacional, tal y como demuestra el incremento de las demandas de asesoramiento de mercados al Instituto Municipal de Mercados de Barcelona, que desde sus inicios ha impulsado la modernización y remodelación de los mercados barceloneses con vistas a adaptar la oferta comercial y las infraestructuras a las demandas de la ciudadanía, así como en el liderato de Barcelona de la Red Europea de 
Mercados Emporion, la entrada de la red de mercados municipales de Barcelona en la Unión Mundial de Mercados (WUWM) y el papel puntero de Mercados de Barcelona en el proyecto europeo Med Emporion.

Otro ejemplo significativo lo encontramos en el caso de la ciudad de Vigo, que cuenta con una estrategia integral dirigida a obtener inversiones privadas para estas instalaciones a las que el Ayuntamiento no puede llegar por los costes elevados de las mismas, al tiempo que se persigue mayor exigencia en la prestación eficaz de los servicios con medios materiales y personales y favorecer la existencia de actividades más allá de la venta de productos alimenticios de primera necesidad. A todo ello se añade la reordenación física de los puestos así como de los elementos comunes de los edificios, mediante la renovación del mobiliario, la mejora del aspecto físico del interior y exterior de los mercados y la incorporación de una cadena de supermercados en algunos de los centros como oferta complementaria de alimentación.

\section{DEBATE}

Tras el análisis efectuado es posible identificar aquellos aspectos de las actuaciones estudiadas que plantean dudas o interrogantes y que los hacen merecedores de un análisis crítico. Estos aspectos se centran en la figura de los Mercados Municipales y la legitimidad de su redefinición en el contexto económico actual; la licitud de la desafectación del dominio público como mecanismo de financiación de las operaciones de revitalización urbana de espacios dotacionales; la operatividad de la aplicación de nuevas figuras como el complejo inmobiliario y la precisión en la naturaleza jurídica de las operaciones proyectadas.

a) El valor de los Mercados Municipales en la política de regeneración urbana del Ayuntamiento de Madrid.

Tal y como ya se ha quedado patente, se detecta en ambas operaciones la presencia de un factor innovador y peculiar, los Mercados municipales, muy característico de los barrios del centro urbano de Madrid que pasa a convertirse en el elemento catalizador esencial para materialización de las mismas

Este renovado valor de los Mercados Públicos y sus posibilidades para la potenciar los aspectos sociales, urbanos, culturales y económicos de un ámbito, ha tenido, en el caso de Madrid, dos precedentes claros: El Mercado de San Antón y el Mercado de San Miguel, a pesar de que ambos casos presentaban el rasgo 
distintivo de ser edificios de uso dotacional de titularidad privada. En ambos casos, y bajo un principio de salvaguarda de los valores y características funcionales y comerciales que, han convertido a los mismos en piezas señeras de calidad y prestigio a nivel de distrito o barrio, se inaugura un nuevo modelo en el que es posible la flexibilidad (horarios, disposición, oferta de productos, modalidades de consumo...) y la mezcla de usos superando la mera provisión de productos alimentarios. Se trata de locales que ofrecen productos menos cotidianos, como ropa, calzado, papelerías, librerías, calzado electrodoméstico, mobiliario o menaje del hogar, todos ellos imprescindibles pero que con frecuencia se han descentralizado, alejándolos del centro urbano.

Frente a los comercios minoristas que se dispersan en el tejido urbano, los Mercados Municipales, como comercios de centralidad, se sitúan en los ejes principales de la ciudad que conectan con otros sistemas externos. Sobre ellos y su potencial el Informe, de la Coordinadora General de Urbanismo de 23 de abril de 2010, sobre los planteamientos a desarrollar para impulsar la competitividad de los Mercados Municipales, ya aludió al objetivo de crear un nuevo marco normativo que permitiera dar solución a las dificultades actuales de los Mercados municipales, mejorando la competitividad mediante el impulso municipal de los mismos. Para la consecución de este objetivo, el informe planteó dos posibilidades: La vía de las modificaciones del PGOUM para el cambio de la calificación de los mercados como dotacionales de Servicios Públicos como Servicios Terciarios o, la modificación de la Ordenanza de Mercados Municipales y la normativa del PGOUM para clarificar el alcance del uso de servicio público de mercado, estableciendo un régimen flexible de usos asociados que pueden llegar a implantarse en los mismos. En esta línea, resulta también relevante el pronunciamiento de la Comisión de Seguimiento e Interpretación del PGOUM, acuerdo de 10 de junio de 2010, el cual realizando una interpretación integrada del artículo 7.11.1 de las NNUU (definición de los mercados como uso dotacional de servicios colectivos como instalaciones para proveer de productos alimentarios para el abastecimiento de la población) con la definición que del mismo establece la normativa de régimen local (abastecimiento de artículos de consumo de primera necesidad), concluye que el mercado no reduce su actividad al abastecimiento de alimentos sino que ha de entenderse ésta extendida a los artículos de consumo de todo tipo. Huelga decir, que esta redefinición en el enfoque y en la actividad de los Mercados Municipales supone en definitiva, crear las condiciones adecuadas para hacer atractiva a la iniciativa privada la gestión y explotación de los mismos lo que, necesariamente nos lleva al clásico debate que se planteó en su día respecto de las instalaciones deportivas municipales, del riesgo de privatización encubierta o 
de facto de lo que legalmente se configura como servicios públicos destinados a la totalidad de los ciudadanos con independencia de su poder adquisitivo o circunstancias sociales.

Esta cuestión nos aproxima a la problemática, que se aborda en el siguiente apartado, de la pérdida de dotaciones de servicio colectivo para la ciudad y la obligación de compensar por imperativo legal, el incremento de usos lucrativos,

\section{b) Desafectación de dominio público.}

Estrechamente vinculado a la cuestión anterior, aunque con entidad propia, nos encontramos con la decisión, presente en ambas actuaciones, de recalificar parte del uso dotacional de servicios colectivos del mercado, como uso lucrativo en su clase comercial. Esta decisión municipal, y tal y como ponen de relieve, las justificaciones incorporadas en los respectivos estudios económicos-financieros, se motiva en la necesidad de ampliar la oferta de actividades y servicios a los ciudadanos cuya demanda responde, en gran medida, a hábitos consumistas, pero desde luego pone al descubierto la necesidad de contar con la inversión privada como única forma de garantizar el éxito de estas operaciones, paradójicamente, en espacios dotacionales.

Queda pues patente cómo las operaciones se sustentan en la opción de incorporar edificabilidad lucrativa del uso terciario, en detrimento del uso dotacional para posibilitar su enajenación a particulares. En otras palabras, la viabilidad de la actuación se apoya básicamente en la desafectación de usos dotacionales para dar entrada a la financiación privada. Aunque es cierto que esta alternativa se ve compensada por exigencia legal, con el aumento proporcional de los otros usos dotacionales públicos (zona verde, equipamiento), también lo es que este planteamiento supone un cambio radical en la concepción del modelo urbano basado hasta este momento, al menos en apariencia, en la conservación de lo público, en el incremento de la oferta de usos dotacionales y en la intervención unilateral de la Administración en operaciones dotacionales de recuperación urbana.

Se constata con esta decisión un importante cambio en el modelo de la calidad urbana, que pasa de residenciar ésta en la existencia y defensa de usos públicos, al servicio potencial de todos los ciudadanos, a la proliferación y fomento de usos terciarios más vinculados a un segmento poblacional con mayores recursos económicos que asocian el tiempo de ocio con la actividad de consumo. Se insiste en cómo esta opción municipal pone de manifiesto la necesidad de acudir a usos lucrativos rentables para la iniciativa privada como mecanismo de financia- 
ción de las actuaciones de revitalización, así y ante la insuficiencia/inexistencia de la inversión pública se hace preciso contar con la inversión privada orienta, como es lícito, al beneficio. Resulta también curioso comprobar cómo la respuesta municipal a las alegaciones formuladas en este sentido se centra en justificar la existencia de una posibilidad legal por la que un bien de dominio público puede pasar, tras la correspondiente tramitación administrativa, a ser patrimonial y en consecuencia a ser enajenado, pero obvia el problema de fondo que es que resulte plenamente justificado y motivado a la luz del interés público la desafectación de lo público.

c) El complejo inmobiliario como mecanismo de compensación dotacional en ámbitos de suelo urbano consolidado.

En el caso del Mercado de la Cebada, se acude de forma novedosa al complejo inmobiliario, nueva figura jurídica que supera la tradicional concepción, con fundamento en el Derecho Romano, de que el propietario del suelo lo es también de vuelo y del subsuelo. Esta figura está regulada en el artículo 17 del Texto Refundido de la Ley del Suelo de 2008, modificado recientemente por la Ley $8 / 2013$ de rehabilitación, regeneración y renovación urbanas.

La utilización práctica de esta nueva figura legal posibilita la convivencia en la misma parcela de un dominio público en superficie y una propiedad privada en el subsuelo, previa desafectación del mismo para su conversión como finca especial de atribución privativa, con ciertas limitaciones y servidumbres. En el caso del Mercado de la Cebada, su uso permite la localización del garaje-aparcamiento bajo la rasante de parte del viario público y la zona verde, superponiéndose ambas calificaciones urbanísticas dotando de flexibilidad al ámbito. Asimismo, el complejo inmobiliario se plantea en el caso del Mercado de la Cebada, como forma de obtener más superficie de suelo en ámbitos consolidados y colmatados, a efectos de localizar las superficies de usos dotacionales exigidas por ley para compensar el equilibrio con los nuevos usos lucrativos.

No obstante, y a pesar de tratarse de una posibilidad admitida legalmente no deja de plantear el mismo interrogante en torno a la licitud de la desafectación del dominio público para su enajenación y obtención de ingresos privados por parte del Ayuntamiento (RAmírez, 2010). También y por lo que respecta a su uso como técnica compensatoria de las dotaciones, podría resultar cuestionable el efecto que produce en cuanto al mantenimiento, no tanto de la cantidad, como de la calidad y funcionalidad de las dotaciones. Sobre este punto hay que recordar que la exigencia de la LSCM artículo 67 exige medidas compensatorias precisas 
para mantener la cantidad y calidad de las dotaciones previstas respecto del aprovechamiento urbanístico del suelo, sin incrementar éste en detrimento de la proporción ya alcanzada entre unas y otro y, en todo caso, asegurar la funcionalidad y el disfrute del sistema de redes de infraestructuras, equipamientos y servicios públicos. En este sentido bien podría esgrimirse que la disposición de zonas verdes o espacios públicos en las cubiertas de edificios merman la funcionalidad y por lo tanto la calidad de las mismas, al complicar su acceso y desde luego modificar las condiciones normales para su uso y disfrute por parte de los ciudadanos.

d) Naturaleza jurídica de las actuaciones de revitalización analizadas.

La calificación jurídica de la actuación no es un tema menor por cuanto que permite identificar los objetivos básicos a realizar así cómo la legalidad y pertinencia de las decisiones adoptadas para la ordenación y gestión de los ámbitos. A la luz de la regulación contenida en el artículo 14 del texto Refundido de la Ley del Suelo de 2008, dentro de las actuaciones de transformación urbanística cabe distinguir entre las de nueva urbanización, las de reforma o renovación y las de dotación.

Si bien las primeras son claramente identificables, por corresponderse con operaciones de creación de ciudad y transformación de suelos, las otras dos categorías precisan de mayor discernimiento. Doctrinalmente, las actuaciones de reforma o renovación, con independencia de que tengan lugar en suelos urbanos consolidados o no consolidados, tienen como objetivo reformar y renovar la urbanización de un modo integral, innovador, yendo por lo tanto más allá de la mera obtención de dotaciones (IgLesias,2004). Por su parte las actuaciones de dotación no provocan esta renovación integral de la urbanización sino que producen efectos de un modo más parcial y concreto.

Conforme a estas consideraciones, las actuaciones seguidas en el Mercado de Barceló y en el Mercado de la Cebada bien podría considerarse como actuaciones de reforma y renovación urbana ya que si bien a través de las mismas se obtienen dotaciones, lo cierto es que éstas se dirigen a compensar el incremento de otros usos lucrativos al tiempo que suponen una renovación integral en la urbanización de un ámbito relevante.

e) Colaboración institucional y participación vecinal 
En el caso de las actuaciones analizadas se trasluce una mínima participación vecinal durante el proceso de su diseño, planificación y proyecto, en el sentido de que la misma se reduce a las alegaciones efectuadas por los particulares en los preceptivos trámites de información pública, a diferencia de otras actuaciones municipales, que bien podrían también considerarse de regeneración y recuperación urbana, como el proyecto singular del Eje Prado-Recoletos o Madrid-Río, en los que el Ayuntamiento acercó a los ciudadanos sus propuestas, desde la fase inicial de concepción de los mismos, a través de avances no exigidos legalmente y de exposiciones con acceso público.

Ciertamente se trata de actuaciones muy endógenas, en las que no se produce la participación de otras instituciones ni otras Administraciones Públicas. Sobre este aspecto es reseñable también cómo el Ayuntamiento opta, en ambos casos, por remitir la ordenación pormenorizada a la figura del plan especial, cuyo procedimiento de tramitación y aprobación queda sustraído por completo a cualquier tipo de control autonómico. La única participación que se propicia es a nivel del proyecto a través del concurso de ideas que se convoca en ambas actuaciones. Llama la atención cómo la opción que se hace desde el propio Plan General, es ajustar la ordenación pormenorizada de los respectivos planes especiales al resultado de los proyectos ganadores. De un modo indirecto, se deja en manos de la iniciativa privada el contenido del posterior planeamiento de desarrollo, lo que genera ciertas dudas de coherencia legal a la vista de la anulación que se efectuó en su día por los tribunales de justicia, de la figura de los Convenios de planeamiento al objeto, precisamente, de evitar sacar de la esfera de decisión municipal el contenido de la potestad de planeamiento, indisponible, en cuanto potestad pública.

\section{PARTE PROPOSITIVA}

Las reflexiones efectuadas sobre las circunstancias y la problemática en torno a las cuales se han planteado las actuaciones de revitalización de espacios públicos rotacionales analizadas, permiten apuntar algunas propuestas que se considera sería útil para el futuro y el éxito de este tipo de intervenciones públicas.

La primera de ellas es la necesidad de afrontar las actuaciones de revitalización de espacios públicos como algo integral en las que queden englobadas no sólo las actuaciones sobre las infraestructuras y edificios públicos sino también los edificios privados incluidos en el ámbito, cómo fórmula para conseguir un impacto global en la imagen de la ciudad y, al mismo tiempo, como forma de in- 
volucrar a los vecinos de cada ámbito en las estrategias públicas de recuperación de espacios dotacionales.

Por otra parte, se estima preciso la búsqueda de acciones coordinadas desde la fase inicial del diseño de las actuaciones, debiéndose propiciar por la Administración municipal la intervención de otras Administraciones Públicas, de instituciones o entidades públicas o privadas y de los propios ciudadanos. Este planteamiento, provocaría mayor complicidad, compromiso y consenso sobre las decisiones públicas de rehabilitación urbana lo que, en última instancia garantizaría el éxito de las mismas. La participación de otras instancias públicas, mediante figuras como el consorcio o los convenios, podría facilitar la gestión de las operaciones desde el punto de vista monetario de la financiación como el de las permutas de suelo, los derechos de superficie las cesiones de uso, etc...

Finalmente, se consideran oportunas todas las modificaciones normativas, en la línea de la reciente Ley $8 / 2013$, que creen un marco preciso, concreto y claro sobre las figuras que pueden servir para flexibilizar las operaciones de recuperación urbana.

\section{CONCLUSIONES GENERALES}

Las acciones de revitalización de espacios públicos dotacionales se configuran, en el momento actual como parte irrenunciable de las políticas urbanas municipales, tanto como mecanismo de recuperación y regeneración de la ciudad en sí mismo, indisolublemente unido a los fines públicos asociados a la potestad pública en materia de urbanismo, como vía de recuperación e impulso social y económico de la ciudad.

Si bien se trata de actuaciones coyunturales referidas a espacios con identidad propia dentro de los cascos urbanos, lo cierto es que presentan una cierta vocación estructural al incidir de modo conjunto en el resultado final de la imagen de la ciudad y su evolución social, económica, cultural, turística y laboral. A través de este tipo de intervenciones se pone de relieve el carácter dinámico de la ciudad, del urbanismo y del planeamiento al suponer un rediseño de la ciudad ya construida y urbanizada, frente a planteamientos más estáticos que abogan por una mera conservación del patrimonio de las ciudades.

En todas ellas cabe resaltar su gran complejidad y dificultad en comparación con las operaciones de creación de ciudad y expansión urbana, ya que al proyectarse sobre ámbitos de suelo urbano ya consolidados y agotados, están marcadas por una serie de condicionantes y limitaciones muy específicas no ex- 
trapolables a las operaciones de transformación urbanística. Esta circunstancia permite valorar de un modo muy positivo impulsos legislativos como el realizado por la Ley 8/2013 de rehabilitación, regeneración y renovación urbanas, que pretender remover los obstáculos legales existentes para poner en práctica este tipo de operaciones, garantizar su viabilidad técnica y económica y reforzar su concepción como actuaciones integrales.

\section{BIBLIOGRAFÍA}

Hervás Más, J. (2009): "Las actuaciones de dotación y renovación en el suelo urbanizado. La Regeneración de la Ciudad", El consultor de los Ayuntamientos y de los Juzgados, $\mathrm{n}^{\circ}$ 5, Quincena 15-29 Marzo. ref. 732/2009, Tomo I. 732 p.

Iglesias Calvo, A. (2004): "Las actuaciones urbanísticas y de Vivienda en la revitalización de centros tradicionales", El Agente Rehabilitador. Thomsom-Aranzadi. Noviembre 2004.

Ramírez Sánchez, J. M. (2010): "La gestión del suelo urbanizado: Las actuaciones de dotación en el Texto Refundido de la Ley del Suelo", Revista Jurídica de Navarra, ${ }^{\circ}$ 49, Enero-Junio, 185-214.

\section{Expedientes consultados:}

Memoria del Plan General de Ordenación Urbana de Madrid, aprobado por Acuerdo

Memorias de Gestión del Área de Gobierno de Urbanismo y Vivienda. Años 2008-20092010-2011

Expediente $\mathrm{n}^{\circ} 711 / 2008 / 03814$. Mercado Barceló.

Expediente $n^{\circ} 711 / 2010 / 26333$. Mercado de la Cebada. APE 01.07 "Plaza de la CebadaCarrera de San Francisco". 